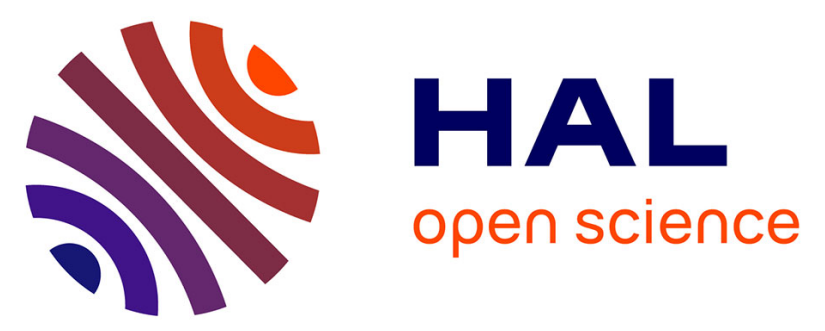

\title{
Assessment of the potential activity of major dietary compounds as selective estrogen receptor modulators in two distinct cell models for proliferation and differentiation
}

Sylvain Lecomte, Marie Lelong, Gaëlle Bourgine, Théo Efstathiou, Christian Saligaut, Farzad Pakdel

\section{To cite this version:}

Sylvain Lecomte, Marie Lelong, Gaëlle Bourgine, Théo Efstathiou, Christian Saligaut, et al.. Assessment of the potential activity of major dietary compounds as selective estrogen receptor modulators in two distinct cell models for proliferation and differentiation. Toxicology and Applied Pharmacology, 2017, 325, pp.61-70. 10.1016/j.taap.2017.04.005 . hal-01558816

\section{HAL Id: hal-01558816}

\author{
https://hal-univ-rennes1.archives-ouvertes.fr/hal-01558816
}

Submitted on 12 Jul 2017

HAL is a multi-disciplinary open access archive for the deposit and dissemination of scientific research documents, whether they are published or not. The documents may come from teaching and research institutions in France or abroad, or from public or private research centers.
L'archive ouverte pluridisciplinaire HAL, est destinée au dépôt et à la diffusion de documents scientifiques de niveau recherche, publiés ou non, émanant des établissements d'enseignement et de recherche français ou étrangers, des laboratoires publics ou privés. 
Assessment of the potential activity of major dietary compounds as selective estrogen receptor modulators in two distinct cell models for proliferation and differentiation

Sylvain Lecomte ${ }^{1}$, Marie Lelong ${ }^{1}$, Gaëlle Bourgine ${ }^{1}$, Theo Efstathiou ${ }^{2}$, Christian Saligaut ${ }^{1}$, Farzad Pakdel $^{1 \#}$

${ }^{1}$ Institut de Recherche en Santé-Environnement-Travail (IRSET), Inserm UMR 1085, Team Transcription, Environment and Cancer, University of Rennes 1. 9 Avenue du Pr Léon Bernard, 35000 Rennes, France

${ }^{2}$ Laboratoire Nutrinov, Technopole Atalante Champeaux, 8 rue Jules Maillard de la Gournerie, 35012 Rennes Cedex, France

"Corresponding author: Dr. Farzad Pakdel, farzad.pakdel@univ-rennes1.fr, Phone number: +33223235132, Fax number: +33223235055 


\section{Abstract}

Estrogen receptors (ERs) $\alpha$ and $\beta$ are distributed in most tissues of women and men. ERs are bound by estradiol (E2), a natural hormone, and mediate the pleiotropic and tissue-specific effects of E2, such as proliferation of breast epithelial cells or protection and differentiation of neuronal cells. Numerous environmental molecules, called endocrine disrupting compounds, also interact with ERs. Phytoestrogens belong to this large family and are considered potent therapeutic molecules that act through their selective estrogen receptor modulator (SERM) activity. Using breast cancer cell lines as a model of estrogen-dependent proliferation and a stably ER-expressing PC12 cell line as a model of neuronal differentiating cells, we studied the SERM activity of major dietary compounds, such as apigenin, liquiritigenin, daidzein, genistein, coumestrol, resveratrol and zearalenone. The ability of these compounds to induce ER-transactivation and breast cancer cell proliferation and enhance Nerve Growth Factor (NGF) -induced neuritogenesis was assessed. Surprisingly, although all compounds were able to activate the ER through an estrogen responsive element reporter gene, they showed differential activity toward proliferation or differentiation. Apigenin and resveratrol showed a partial or no proliferative effect on breast cancer cells but fully contributed to the neuritogenesis effect of NGF. However, daidzein and zearalenone showed full effects on cellular proliferation but did not induce cellular differentiation. In summary, our results suggest that the therapeutic potential of phytoestrogens can diverge depending on the molecule and the phenotype considered. Hence, apigenin and resveratrol might be used in the development of therapeutics for breast cancer and brain diseases.

Keywords: Phytoestrogens, SERM, breast cancer cell lines, PC12, proliferation, differentiation

\section{Highlights}

- SERM activity of dietary compounds on proliferation and differentiation is studied.

- All the dietary compounds tested transactivate estrogen receptors. 
- Apigenin and resveratrol could be good candidates for future therapeutics.

- Daidzein and zearalenone are to be avoided to maintain human health. 


\section{Introduction}

Estrogen receptors (ERs) belong to the nuclear receptor superfamily and are coded by two different genes (ER $\alpha$ and $E R \beta$ ) located on two different chromosomes. ERs are distributed in main tissues of organisms, in women and men, but the ratio between ER $\alpha$ and ER $\beta$ may differ in different tissues. ER $\alpha$ is considered the more potent regulator of gene expression, while ER $\beta$, when it is coexpressed with $E R \alpha$, appears to have a restraining effect on $E R \alpha$ activities (Jiang et al., 2013; Ström et al., 2004). Thus, depending on the tissue, ERs could have different effects, such as proliferation or differentiation. These pleiotropic effects can be obtained with the same molecule, thus defining the term selective estrogen receptor modulator (SERM). Indeed, in addition to estradiol (E2), ERs can bind several environmental compounds, including the so-called xenoestrogens that belong to the family of endocrine disruptors. These molecules can act on ERs selectively, either as agonists or as antagonists, which allows them to stimulate or inhibit estrogenic responses selectively depending on the target tissue. Therefore, xenoestrogen exposure could affect different organs differently based on the distribution and the isoform of the ERs present and the transcriptional co-regulators that interact with ERs (Diamanti-Kandarakis et al., 2009; Kerdivel et al., 2013). Currently, SERM compounds are often studied to develop new therapies against various pathologies, such as the hormone-dependent cancers, while maintaining the protective effect of estrogens on the brain or on bones for example. In this work, we studied the SERM activity of major dietary compounds that belong to different chemical classes and that have previously been noted to bind ERs. These natural chemicals could represent molecule candidates that are safer than synthetic molecules for the treatment of estrogen-related pathologies. Among them we studied the two isoflavones, daidzein and genistein, found in soy (Gong et al., 2014; Li et al., 2013; Nikov et al., 2000); the flavones apigenin found in parsley or chamomile (Li et al., 2013); and liquiritigenin found in licorice root (Gong et al., 2014). Another chemical class of phytoestrogens is represented by coumestrol, a coumestan also found in soy and alfalfa (Li et al., 2013; Nikov et al., 2000). Resveratrol is a stilbene found in 
grapes and red wine that interacts with ERs, in addition to its antioxidant activity (Chen and Chien, 2014). Finally, zearalenone a mycotoxin found in poorly stored cereals and present in $15 \%$ of cereals consumed in Europe (EFSA Panel on Contaminants in the Food Chain, 2011) is described as a potent estrogenic compound ( $\mathrm{Li}$ et al., 2012; Nikov et al., 2000). The plasma concentration of phytoestrogens differs greatly in the human population. For instance, the mean genistein plasma concentration is found to be between $0.5 \mathrm{nM}$ in Finnish males and $276 \mathrm{nM}$ in Japanese males. However, after intake of dietary phytoestrogen, a plasma peak is observed that could reach a concentration of 0.2 to $6.5 \mu \mathrm{M}$ with a bioavailability between $5 \%$ to $66 \%$ (Whitten and Patisaul, 2001). In this study, we first aimed to confirm the ability of various dietary compounds to induce ER $\alpha$ transactivation at concentrations that can be found in organisms. Second, we aimed to evaluate their potent proliferative and differentiating effects. The following three cellular models were used: MCF7 and T47D cells, two ER-positive and E2-dependent proliferative breast cancer cell lines, and PC12 cells stably transfected with ER $\alpha$ that are able to develop a pseudoneuronal phenotype (neurite outgrowth) when treated with nerve growth factor (NGF). We previously established that the expression of ER $\alpha$ potentiates the effect of NGF on neurite outgrowth in the presence of E2 in PC12 cells through non genomic pathway (Habauzit et al., 2011; Mérot et al., 2009). Indeed in cells ER $\alpha$ acts through two pathways named genomic pathway that involves DNA-binding and non-genomic pathway that involves modulation of intracellular pathway (MAPK and PI3K/AKT) by plasma membrane anchored ER (Levin and Pietras, 2008). This study is the first study, to the best of our knowledge that directly compared the activity of several dietary compounds using two distinct ERregulated phenotypes.

In summary, among the molecules that we tested, four showed differential effects depending on the proliferation or the differentiation phenotypes. Apigenin and resveratrol appeared to be the most promising compounds, which showed a partial agonistic activity, inducing slight cellular proliferation, but behaved as full agonists in neuritogenesis. In contrast, the results obtained with zearalenone confirmed its deleterious effect, with a full agonist effect regarding cellular proliferation, 
and it was not able to induce the differentiation of PC12-ER cells. Finally, daidzein, similar to zearalenone, presented a full agonist effect regarding cellular proliferation and an absence of effect on differentiation. This characterization is a first step in determining the therapeutic potential of dietary compounds on breast cancer and on brain diseases.

\section{Materials and methods}

\section{Cell culture and reagents}

MCF-7 cells were grown in DMEM (4.5 g/L glucose) supplemented with non-essential amino acids (NEAA) (Invitrogen, Waltham, MA, USA) and $10 \%$ fetal bovine serum (FBS) (Biowest, France). T47D cells were maintained in RPMI 1640 supplemented with NEAA, sodium pyruvate (Invitrogen, Waltham, MA, USA) and $10 \%$ FBS (Biowest, France). PC12 cells stably transfected with ER $\alpha$ (PC12-ER) or with the empty vector (PC12-Cont) were cultured as described previously (Habauzit et al., 2014). Cells were grown with penicillin/streptomycin at $37^{\circ} \mathrm{C}$ in $5 \% \mathrm{CO}_{2}$. For treatments, all cell lines were cultured at least $24 \mathrm{~h}$ in red phenol-free DMEM supplemented with $2.5 \%$ charcoal stripped FBS for MCF-7 and PC12 cells and $5 \%$ for T47D. All drugs used in this work were purchased from SigmaAldrich (France) and are presented in table 1.

\section{Luciferase assay}

Cells (30,000 cells/well for MCF-7 and 40,000 cells/wells for T47D and PC12-ER) were plated in 24well plates. After serum and steroid deprivation, cells were transfected overnight by $100 \mathrm{ng}$ of estrogen responsive element-thymidine kinase (ERE)-TK-luciferase vector, which encodes luciferase under the control of one ERE, and by $20 \mathrm{ng}$ for MCF-7, $100 \mathrm{ng}$ for T47D and $150 \mathrm{ng}$ for PC12 of CMV- $\beta$ galactosidase vector, which serves as a transfection efficiency control, using JetPEl (Polyplus transfection, France). Next, cells were treated with different doses of E2 or different compounds. Cells were lysed in Passive Lysis Buffer (Promega, Madison, WI, USA), and luciferase activity was determined using a luciferase assay system (Promega, Madison, WI, USA). 


\section{Proliferation assay}

Cells (20,000 cells/well for MCF-7 and 40,000 cells/well for T47D) were plated in 24-well plates. Then, cells were deprived of steroids and serum for $72 \mathrm{~h}$. Cells were treated with solvent (ethanol and DMSO) as a negative control, with different doses of E2 or with different doses of phytoestrogens for 6 days with a renewed treatment at day 3. After treatment, cells were trypsinized, and cell number was determined with a TC10 Automated Cell Counter (BioRad, Hercules, CA, USA).

RNA extraction and real-time $P C R$

Cells (250,000 cells/well for MCF-7 and 400,000 cells/well for T47D) were plated in 6-well plates. After $30 \mathrm{~h}$ of serum and steroid deprivation, cells were treated with solvent (ethanol and DMSO) as a control or with different doses of E2 or dietary compounds. Total RNAs were extracted with the RNeasy mini kit (Qiagen, Germany), according to the manufacturer's instructions. Then, total RNAs were reverse transcribed using an M-MLV RT kit (Invitrogen, Waltham, MA, USA) according to the manufacturer's instructions. For real-time PCR, $5 \mathrm{ng}$ of CDNA was used with $150 \mathrm{nM}$ primers (CXCL12: forward primer CACCATTGAGAGGTCGGAAG, and reverse primer AATGAGACCCGTCTTTGCAG; ESR1/ERa: forward primer TTTATGGGAAAAGGCTCAAA, and reverse primer GACAAAACCGAGTCACATCA; ESR2/ERß: forward primer AGAGTCCCTGGTGTGAAGCAAG, and reverse primer GACAGCGCAGAAGTGAGCATC; GAPDH: forward primer GGGCATCCTGGGCTACACTG, and reverse primer GGGCATCCTGGGCTACACTG) and with the iTaq Universal SYBR Green supermix (BioRad, Hercules, CA, USA). Real-time PCR was performed on a MyiQ apparatus, and results were analyzed with iQ5 software (BioRad, Hercules, CA, USA).

\section{Determination of PC12-ER neurite outgrowth}

Briefly, 15,000 cells/well were plated in 12 -well plates. After $48 \mathrm{~h}$, cells were grown in serum and steroid deprivation medium. Then, cells were treated with NGF at $5 \mathrm{ng} / \mathrm{mL}$ alone or combined with 
dietary compounds at different doses for $72 \mathrm{~h}$. The differentiation state of PC12 was scored as previously described (Habauzit et al., 2014; Mérot et al., 2009).

\section{Results}

Effect of dietary compounds on ER activation in ER-positive breast cancer cell lines

We first assessed the expression ratio between ER $\alpha$ and ER $\beta$ in MCF-7 and T47D cells by real time PCR (Fig 1A). In MCF-7 cells, ER $\alpha$ is found 8 times more expressed than ER $\beta$. Although, ER $\beta$ mRNA is weakly expressed, it is detectable by real time PCR assay with an average of $\mathrm{Ct}$ at 32.9. As expected, $E R \alpha / E R \beta$ ratio was clearly different in T47D cells. As shown in Fig 1A, ER $\alpha$ mRNA level was found only 1.8 times higher than ER $\beta$ mRNA.

To study the activation of ERs by dietary compounds, MCF-7 (Fig 1B) and T47D (Fig 1C) cells were transfected with a luciferase reporter plasmid under the control of an estrogen responsive element (ERE). The natural hormone, E2 showed a maximum luciferase activity at $10^{-8} \mathrm{M}$ in MCF-7 and T47D cells compared to the solvent (ethanol and DMSO), which was used as a negative control. The dietary compounds tested were able to activate ER, as shown by the increase in luciferase activity in the two cell lines. Although luciferase activity presented a few differences in terms of intensity, the profile between the two cell lines was quite similar, except for cells treated with resveratrol. Indeed, in MCF7 cells, the maximum luciferase activity was reached at $10^{-6} \mathrm{M}$ resveratrol, while it was reached at $10^{-}$

${ }^{5} \mathrm{M}$ in T47D cells. Unlike zearalenone, the other compounds require a concentration $100-1000$ times greater than that of E2 to stimulate luciferase activity. Zearalenone induced luciferase activity at $10^{-9} \mathrm{M}$ in MCF-7 cells, a concentration equivalent of the plasma concentration of E2. At high doses, luciferase activity decreased by 2-fold, which could be explained by the occurrence of cellular stress that could affect luciferase stability.

Effect of dietary compounds on the proliferation of ER-positive breast cancer cell lines 
To test the SERM activities of selected compounds, their effects on proliferation (Fig 2) of the two ERpositive breast cancer cell lines (MCF-7 and T47D) were assessed. After treatment, cells were counted, and the results were expressed as percentage of $10^{-9} \mathrm{M} E 2$ effect. These two cell lines have an E2-dependent proliferation. As shown in figure 2, E2 at $10^{-9} \mathrm{M}$ for MCF-7 cells and $10^{-8} \mathrm{M}$ for T47D cells induced strong proliferation of cells, reaching a maximum of 4.5 times more than control cells. In MCF-7 cells, liquiritigenin (Fig 2A), daidzein, genistein (Fig 2B) and coumestrol (Fig 2C) induced proliferation as well as E2 but at a concentration 1000 - 10000 times higher. In contrast, zearalenone (Fig 2C) showed powerful estrogenic activity by stimulating cell proliferation starting at $10^{-10} \mathrm{M}$ to a maximum at $10^{-8} \mathrm{M}$. However, zearalenone showed clear toxic effects on cell proliferation at $10^{-5} \mathrm{M}$ (Fig $2 \mathrm{C}$ and 2F). Apigenin increased cell numbers slightly, which reached approximately $50 \%$ of the E2 $10^{-9} \mathrm{M}$ proliferation rate. Finally, resveratrol did not induce proliferation at any of the doses tested. One-way ANOVA with a Bonferroni's multiple comparison post hoc test, revealed that only resveratrol was significantly different from E2 treatment $(p<0.05)$. In T47D cells, the proliferative effects of liquiritigenin (Fig 2D), daidzein (Fig 2E), coumestrol (Fig 2F) and resveratrol were quite similar to that observed in MCF-7 cells. However, the proliferative effect of Apigenin (Fig 2D) reached $80 \%$ of the E2 effect. At the highest concentration tested $\left(10^{-5} \mathrm{M}\right)$, cytotoxic events occurred with apigenin (Fig 2D) and genistein (Fig 2E). Zearalenone (Fig 2F) also induced strong proliferation of T47D cells but showed less toxic effects compared to MCF-7 cells. As in MCF-7 cells, only resveratrol was found to be significantly different from E2 in T47D cells (one-way ANOVA with a Bonferroni's multiple comparison post hoc test, $\mathrm{p}<0.05)$.

\section{Effect of dietary compounds on CXCL12 gene expression}

The chemokine CXCL12 was shown to be linked to proliferation of MCF-7 cells (Boudot et al., 2011). Thus, we used real time PCR to measure CXCL12 gene expression in MCF-7 and also in T47D cells treated with different doses of dietary compounds (Fig 3). The results were expressed as percentage of induction obtained at $10^{-9} \mathrm{M}$ of E2. In MCF-7 (Fig 3A) and T47D cells (Fig 3B), E2 strongly induced 
the expression of CXCL12, reaching a maximum at $10^{-9} \mathrm{M}$ and $10^{-10} \mathrm{M}$ for MCF-7 and T47D cells, respectively. Except for resveratrol, the dietary compounds also induced CXCL12 expression. However, there were slight differences between MCF-7 and T47D cells. For instance, in MCF-7 cells, apigenin strongly induced CXCL12 gene expression but only at $10^{-5} \mathrm{M}$, reaching the same expression level observed with $10^{-9} \mathrm{ME2}(\mathrm{p}<0.001)$. In contrast, in T47D cells, apigenin treatment resulted in a more pronounced dose effect, with induction starting at $10^{-7} \mathrm{M}$ and a significant induction of CXCL12 at $10^{-6} \mathrm{M}$ and $10^{-5} \mathrm{M}(\mathrm{p}<0.05$ and $p<0.01$, respectively). However, the maximum CXCL12 gene expression was $40 \%$ lower than that resulting from E2-stimulation. Liquiritigenin, daidzein, genistein and coumestrol were able to induce CXCL12 expression in MCF-7 cells but only at high concentrations $\left(10^{-6} \mathrm{M}\right.$ and $\left.10^{-5} \mathrm{M}\right)$, while they stimulated CXCL12 expression at lower concentrations $\left(10^{-7} \mathrm{M}\right)$ in T47D cells. Thus, compared to MCF-7 cells, we found that T47D cells were more sensitive to dietary compounds, which may be due to the presence of ER $\beta$. Nevertheless, the results obtained regarding CXCL12 expression are in good agreement with the proliferation test, in which resveratrol is the weakest while zearalenone is the strongest estrogenic and proliferative compound.

\section{Transcriptional activity of dietary compounds in PC12 cells}

The estrogenic activity of dietary compounds on ER $\alpha$ transcriptional activity was evaluated through the same ERE reporter assay described in figure 1. Cells were treated with 3 concentrations of compounds (low: $10^{-8} \mathrm{M}$, medium: $5.10^{-7} \mathrm{M}$ and high: $10^{-5} \mathrm{M}$ or $10^{-11} \mathrm{M}, 5.10^{-10} \mathrm{M}$ and $10^{-8} \mathrm{M}$ for zearalenone). In the PC12 cells stably transfected with ER $\alpha, E 2$ and the dietary compounds apigenin, liquiritigenin, genistein, coumestrol, resveratrol and zearalenone increased the ERE-TK luciferase transcriptional activity compared to cells treated with the solvent (ethanol, DMSO) (Fig 4 A). This increase has been previously demonstrated to be dose-dependent for E2 (Habauzit et al., 2014) and occurred in a dose-dependent manner for the dietary compounds. In contrast, daidzein failed to increase luciferase activity in stably transfected ER cells and then to activate ERE-mediated ER transactivation (Fig 4 A). 
Effect of dietary compounds on the differentiation (neurite outgrowth) of stably transfected ER PC12 cells

To determine the effect of the dietary compounds on differentiation, cells were treated with $5 \mathrm{ng} / \mathrm{ml}$ NGF with or without the compounds. After $72 \mathrm{~h}$ of exposure, the percentage of cells bearing neurite outgrowth was determined by counting (Fig 4B). As described previously (Habauzit et al., 2014; Mérot et al., 2009), treatment with E2 or genistein increased the percentage of NGF-induced differentiated cells compared to NGF alone by approximately $40 \%$. This increase was not significant for $10^{-5} \mathrm{M}$ genistein, due to a high variability and an increase in mortality (Bouron et al., 1999; Habauzit et al., 2014). Interestingly, we also observed $40 \%$ increase in neuritogenesis when cells were treated with apigenin at the three concentrations tested. Concerning liquiritigenin, only the dose at $5.10^{-7} \mathrm{M}$ induced a significantly high percentage of differentiation. Coumestrol induced strong neuritogenesis starting at the first concentration tested, but the effect became non-significant at $10^{-5} \mathrm{M}$. Resveratrol, similar to liquiritigenin, only induced significant neuritogenesis at $5.10^{-7} \mathrm{M}$. Daidzein was unable to activate ER $\alpha$ in PC12-ER cells and, consequently, did not enhance NGFinduced differentiation. Surprisingly, zearalenone was also found to be ineffective in inducing differentiation.

We have identified four compounds, apigenin, daidzein, resveratrol and zearalenone that have differential activity in regard to proliferation and differentiation. To verify that the pro or antidifferentiation effect of these compounds was mediated by ER $\alpha$, control PC12 cells (PC12-Cont) that do not express ER $\alpha$ were used. PC12-Cont were treated with NGF and the four molecules at the most effective concentration that induced a maximum effect on PC12-ER differentiation (Fig 4B in the cartridge). None of these compounds, including E2, were able to increase the NGF-induced neuritogenesis of PC12-Cont cells.

\section{Discussion}


Although the causes of certain diseases associated with estrogen are still unclear, pharmacological research advances have greatly improved the treatment over the last two decades. In particular, compounds that can bind to the estrogen receptor, and thereby activate or inactivate the estrogenic pathways in many diseases such as brain pathologies or breast cancers, can be very promising and may lead to future therapeutics. Toward this goal, and for the first time, using the natural ligand estradiol as a reference and robust cellular tests, we compared the SERM activity of major dietary molecules identified for their ability to bind ER, induce ER transactivation and interact with global cellular mechanisms of proliferation and differentiation. Although the literature about phytoestrogens and breast cancer is abundant, the pro or anti-breast cancer effect of these compounds and the underlying mechanism remain controversial (Leclercq and Jacquot, 2014). First, the effects of E2 and the phytoestrogens on ER transactivation and proliferation were quite similar in MCF7 and T47D cells. It has been reported that most phytoestrogens have a higher affinity for ER $\beta$, except zearalenone, which presents almost the same affinity for ER $\alpha$ and ER $\beta$ (Jiang et al., 2013; Kuiper et al., 1998). Because ER $\alpha$ and ER $\beta$ are present in T47D cells, but ER $\alpha$ is almost exclusive present in MCF7 cells, the proliferative effects of our compounds may be related to ER $\alpha$. The primary difference resided in the bell-shape of the curve observed in the T47D cells at high dose, whereas we observed a plateau in the MCF-7 cells curve. The chemokine CXCL12 is a key factor for proliferation when it is produced by breast cancer cells and for migration and metastasis when it is produced by target tissues (Boudot et al., 2014, 2011). CXCL12 expression is controlled by E2 in MCF-7 and T47D cells. Thus, we used this marker to confirm the proliferative effect of the dietary compounds. Except for zearalenone, which is toxic at high doses, and for resveratrol in T47D cells, the expression of CXCL12 confirmed our proliferation assay.

The effects of polyphenols on neurotrophic pathways are well described. Numerous studies recently described the role of ERK, PI3K/AKT pathways or the antioxidant activity of polyphenols and their effect on neuritogenesis and neuroprotection (Moosavi et al., 2016; Zhao et al., 2015), but the role of ERs in neuritogenesis remains elusive. Nevertheless, E2 effects on neuroprotection after brain injury 
is well documented. For instance, E2 promotes anti-apoptotic events, increases neurogenesis, enhances neurotrophic support and suppresses inflammation (Brown et al., 2009). Among the flavonoids, coumestan, stilbene and mycotoxin analyzed, four compounds presented distinct activity toward proliferation and differentiation. Apigenin and resveratrol showed a slight or no effect on proliferation of breast cancer cell lines, while they had a powerful effect on PC12-ER neuritogenesis. In contrast, daidzein induced breast cancer cell proliferation but did not increase neuritogenesis. Intriguingly, zearalenone, which appeared to be a strong estrogen-mimetic by stimulating ER $\alpha$ transactivation and ER-dependent breast cancer cell proliferation, did not activate PC12-ER differentiation. Many studies have been published about the interaction between these phyto/mycoestrogens and ERs and about the consequences for cells, and some hypotheses can be advanced to explain our observations. First, differences could be linked to the affinity of dietary compounds for ER $\alpha$. Second, the activity of metabolites of the dietary compounds that have been produced during the detoxification process must be considered.

A previous study argued that the affinity of phytoestrogens for ERs was not sufficient to determine their activity because the conformational changes of ERs induced by the ligand could affect the binding of the receptor to its responsive element and its features (Nikov et al., 2000). For instance, a study showed that the gene expression pattern of genistein tended to cluster near E2 while the gene expression pattern of liquiritigenin was very different (Gong et al., 2014). Thus, in this study, we can postulate that, depending on the cellular context, such as breast cancer cells or PC12-ER cells; the presence of $E R \alpha, E R \beta$ or both; and the expression pattern, the cellular fate could be very different between the dietary compounds tested.

Among these compounds, which showed differential effects toward proliferation or differentiation, resveratrol is the most controversial. Its estrogenic or anti-estrogenic activity on breast cancer cell proliferation is not yet clearly determined (Le Corre et al., 2005). Moreover, resveratrol is a type II ligand (angular molecule) that possesses a weak agonistic activity and a possible antagonistic activity (Leclercq and Jacquot, 2014). This type of ligand disturbs the good folding of the helix 12 of ER $\alpha$ after 
it binds and, consequently, can inhibit the full activation of the receptor (Bentrem et al., 2003). This can explain why resveratrol did not induce proliferation in either MCF-7 or T47D cells, despite the weak induction of CXCL12 expression in T47D cells.

Apigenin has a very weak affinity for ER $\alpha$, notably, through a weak interaction with His-524 of the receptor, which is essential for the estrogenic response (Seo et al., 2012). This weak interaction might explain the slight effect of apigenin on transcription at low dose and on breast cancer cell proliferation but does not explain why apigenin increased NGF-induced neuritogenesis of PC12-ER cells even at the lower dose. Moreover, since apigenin did not potentiate NGF-induced neuritogenesis in PC12 control cells, we can conclude that the apigenin effect on PC12 differentiation is mediated by ER $\alpha$. We previously demonstrated that unliganded ER $\alpha$ and E2-liganded ER $\alpha$ act on neuritogenesis according to two opposite mechanisms: an unliganded ER-induced decrease of the cytoplasmic PI3K/AKT signaling pathway associated with a decrease of neuritogenesis and an E2liganded ER which is associated to an increase of neuritogenesis. Interestingly, we showed that these ER actions are DNA-binding independent (Mérot et al., 2009). It has been reported that apigenin acts positively on neurogenesis in adult neural progenitor cells by activating AKT phosphorylation (Taupin, 2009). It is therefore possible that in PC12-ER cells, apigenin as E2 done opposes the ER-dependent inhibition of the PI3K/AKT signaling pathway via non genomic pathway (Levin and Pietras, 2008). The behavior of daidzein was more complex because it induced breast cancer cell proliferation but did not increase neuritogenesis. Daidzein's affinity for ER $\alpha$ was demonstrated to be slightly higher than apigenin (Kuiper et al., 1998) and that could be explain its better activity on transcription and on cell proliferation, as it was demonstrated for genistein compared to apigenin (Seo et al., 2012). However, daidzein was unable to activate ER $\alpha$ transactivation in PC12-ER cells and unable to potentiate PC12-ER cell differentiation. In accordance with our results, a previous study showed that, unlike genistein, daidzein did not increase NGF-induced neuritogenesis in PC12 cells (Miller et al., 1993). Thus, this suggests that daidzein could be rapidly metabolized in PC12 cells, leading to inactive products that do not interact with ER $\alpha$. 
Zearalenone and its derivatives produced during the detoxification phases, interact with ER $\alpha$ and ERß. Zearalenone and some derivatives have been patented as oral contraceptives, but can present harmful effects such as multiple reproductive deficiencies in animals and humans (infertility, constant estrus, reduced litter size, central precocious puberty) (Pazaiti et al., 2012). Zearalenone promotes mammary tumors in mice (Schoental, 1974) and stimulates the growth of breast cancer cell lines (Ahamed et al., 2001). In our study, zearalenone behaves as a full estrogen agonist by inducing strong proliferation, but is not able to potentiate NGF induced neuritogenesis. The synthesis of different zearalenone derivatives, resulting from different cellular metabolic processes in MCF-7, T47D cells, PC12 cells and in vivo models could explain conflicting data. Then, some Zearalenone derivatives have been reported to have high estrogenic potencies and higher that their concentration suggest. For instance, the derivative $\alpha$-zearalenol, resulting from the hydroxylation of zearalenone by hydroxysteroid dehydrogenases (HSDs), was at least in vitro as strong as E2 (Le Guevel and Pakdel, 2001; Shier et al., 2001). The metabolite $\alpha$-zearalanol was shown to improve hippocampal neurogenesis in ovariectomized mice, at the same concentration that E2 (Dong et al., 2014). In our case, zearalenone or its metabolite was accompanied by harmful effects (proliferation of breast cancer cells and an absence of an effect on NGF-induced neuritogenesis). However, further work will be necessary to find the molecular mechanisms involved.

In conclusion, this study evaluated the SERM activity of some major estrogenic dietary compounds by pointing out the role of $E R \alpha$, and predicted differential activities for four compounds with distinct effects toward proliferation or differentiation. However, this study is a precocious screening and furtherwork focusing on molecular mechanisms and the role of various metabolites is necessary. Our data predicted that resveratrol and apigenin might be good candidates for the development of new therapeutics, whereas daidzein and zearalenone should be avoided for breast cancer and neuritogenesis. Nevertheless, it is essential to confirm the abilities of these compounds in animal models using rodents, for instance. Indeed, the antiproliferative effect of botanical estrogens could be tested in nude mice xenograft models, while the neuroprotective and neurotrophic effects 
could be investigated after ischemic injury (Habauzit et al., 2011) or in mice model of Parkinson disease as it was recently reported for puerarin (Zhao et al., 2015).

\section{Acknowledgements}

We kindly thank Frederic Percevault (IRSET, University of Rennes 1 ) for his help with cell culture and Denis Habauzit (IRSET, University of Rennes 1) for his advice and comments on the PC12-ER cell results.

\section{Funding}

This work was funded by Fond Unique Interministériel (FUI, mVolio project), Région Bretagne and Rennes Métropole. This research was also supported by Inserm and CNRS.

\section{Bibliography}

Ahamed, S., Foster, J.S., Bukovsky, A., Wimalasena, J., 2001. Signal transduction through the Ras/Erk pathway is essential for the mycoestrogen zearalenone-induced cell-cycle progression in MCF-7 cells. Mol. Carcinog. 30, 88-98.

Bentrem, D., Fox, J.E., Pearce, S.T., Liu, H., Pappas, S., Kupfer, D., Zapf, J.W., Jordan, V.C., 2003. Distinct molecular conformations of the estrogen receptor al pha complex exploited by environmental estrogens. Cancer Res. 63, 7490-6.

Boudot, A., Kerdivel, G., Habauzit, D., Eeckhoute, J., Le Dily, F., Flouriot, G., Samson, M., Pakdel, F., 2011. Differential estrogen-regulation of CXCL12 chemokine receptors, CXCR4 and CXCR7, contributes to the growth effect of estrogens in breast cancer cells. PLoS One 6, e20898. doi:10.1371/journal.pone.0020898

Boudot, A., Kerdivel, G., Lecomte, S., Flouriot, G., Desille, M., Godey, F., Leveque, J., Tas, P., Le Dréan, Y., Pakdel, F., 2014. COUP-TFI modifies CXCL12 and CXCR4 expression by activating EGF signaling and stimulates breast cancer cell migration. BMC Cancer 14, 407. doi:10.1186/14712407-14-407

Bouron, A., Becker, C., Porzig, H., 1999. Functional expression of voltage -gated $\mathrm{Na}+$ and $\mathrm{Ca} 2+$ channels during neuronal differentiation of PC12 cells with nerve growth factor or forskolin. Naunyn. Schmiedebergs. Arch. Pharmacol. 359, 370-7.

Brown, C.M., Suzuki, S., Jelks, K.A.B., Wise, P.M., 2009. Estradiol is a potent protective, restorative, and trophic factor after brain injury. Semin. Reprod. Med. 27, 240-9. doi:10.1055/s-00291216277

Chen, F.-P., Chien, M.-H., 2014. Phytoestrogens induce differential effects on both normal and malignant human breast cells in vitro. Climacteric 17, 682-91. doi:10.3109/13697137.2014.937688

Diamanti-Kandarakis, E., Bourguignon, J.-P., Giudice, L.C., Hauser, R., Prins, G.S., Soto, A.M., Zoeller, R.T., Gore, A.C., 2009. Endocrine-disrupting chemicals: an Endocrine Society scientific statement. Endocr. Rev. 30, 293-342. doi:10.1210/er.2009-0002 
Dong, Y., Jiang, A., Yang, H., Chen, H., Wang, Y., 2014. Phytoestrogen $\alpha$-zearalanol improves memory impairment and hippocampal neurogenesis in ovariectomized mice. ScientificWorldJournal. 2014, 862019. doi:10.1155/2014/862019

EFSA Panel on Contaminants in the Food Chain, 2011. Scientific Opinion on the risks for publichealth related to the presence of zearalenone in food. EFSA J. 9, 2197. doi:10.2903/j.efsa.2011.2197

Gong, P., Madak-Erdogan, Z., Li, J., Cheng, J., Greenlief, C.M., Helferich, W., Katzenellenbogen, J.A., Katzenellenbogen, B.S., 2014. Transcriptomic analysis identifies gene networks regulated by estrogen receptor $\alpha(E R \alpha)$ and $E R \beta$ that control distinct effects of different botanical estrogens. Nucl. Recept. Signal. 12, e001. doi:10.1621/nrs.12001

Habauzit, D., Ferrière, F., Botherel, N., Flouriot, G., Pakdel, F., Saligaut, C., 2014. Differentiation of PC12 cells expressing estrogen receptor alpha: a new bioassay for endocrine-disrupting chemicals evaluation. Chemosphere112, 240-7. doi:10.1016/j.chemosphere.2014.03.101

Habauzit, D., Flouriot, G., Pakdel, F., Saligaut, C., 2011. Effects of estrogens and endocrine-disrupting chemicals on cell differentiation-survival-proliferation in brain: contributions of neuronal cell lines. J. Toxicol. Environ. Health. B. Crit. Rev. 14, 300-27. doi:10.1080/10937404.2011.578554

Jiang, Y., Gong, P., Madak-Erdogan, Z., Martin, T., Jeyakumar, M., Carlson, K., Khan, I., Smillie, T.J., Chittiboyina, A.G., Rotte, S.C.K., Helferich, W.G., Katzenellenbogen, J.A., Katzenellenbogen, B.S., 2013. Mechanisms enforcing the estrogen receptor $\beta$ selectivity of botanical estrogens. FASEB J. 27, 4406-18. doi:10.1096/fj.13-234617

Kerdivel, G., Habauzit, D., Pakdel, F., 2013. Assessment and molecular actions of endocrine-disrupting chemicals that interfere with estrogen receptor pathways. Int. J. Endocrinol. 2013, 501851. doi:10.1155/2013/501851

Kuiper, G.G., Lemmen, J.G., Carlsson, B., Corton, J.C., Safe, S.H., van der Saag, P.T., van der Burg, B., Gustafsson, J.A., 1998. Interaction of estrogenic chemicals and phytoestrogens with estrogen receptor beta. Endocrinology 139, 4252-63. doi:10.1210/endo.139.10.6216

Le Corre, L., Chalabi, N., Delort, L., Bignon, Y.-J., Bernard-Gallon, D.J., 2005. Resveratrol and breast cancer chemoprevention: molecular mechanisms. Mol. Nutr. Food Res. 49, 462-71. doi:10.1002/mnfr.200400094

Le Guevel, R., Pakdel, F., 2001. Assessment of oestrogenic potency of chemicals used as growth promoter by in-vitro methods. Hum. Reprod. 16, 1030-6.

Leclercq, G., Jacquot, Y., 2014. Interactions of isoflavones and other plant derived estrogens with estrogen receptors for prevention and treatment of breast cancer-considerations concerning related efficacy and safety. J. Steroid Biochem. Mol. Biol. 139, 237-44. doi:10.1016/j.jsbmb.2012.12.010

Levin, E.R., Pietras, R.J., 2008. Estrogen receptors outside the nucleus in breast cancer. Breast Cancer Res. Treat. 108, 351-61. doi:10.1007/s10549-007-9618-4

Li, Y., Burns, K.A., Arao, Y., Luh, C.J., Korach, K.S., 2012. Differential estrogenic actions of endocrine disrupting chemicals bisphenol A, bisphenol AF, and zearalenone through estrogen receptor $\alpha$ and $\beta$ in vitro. Environ. Health Perspect. 120, 1029-35. doi:10.1289/ehp.1104689

Li, Y., Luh, C.J., Burns, K.A., Arao, Y., Jiang, Z., Teng, C.T., Tice, R.R., Korach, K.S., 2013. Endocrine Disrupting Chemicals (EDCs): In Vitro Mechanism of Estrogenic Activation and Differential Effects on ER Target Genes. Environ. Health Perspect. 121, 459-66. doi:10.1289/ehp.1205951

Mérot, Y., Ferrière, F., Gailhouste, L., Huet, G., Percevault, F., Saligaut, C., Flouriot, G., 2009. Different outcomes of unliganded and liganded estrogen receptor-alpha on neurite outgrowth in PC12 cells. Endocrinology 150, 200-11. doi:10.1210/en.2008-0449

Miller, D.R., Lee, G.M., Maness, P.F., 1993. Increased neurite outgrowth induced by inhibition of protein tyrosine kinase activity in PC12 pheochromocytoma cells. J. Neurochem. 60, 2134-44.

Moosavi, F., Hosseini, R., Saso, L., Firuzi, O., 2016. Modulation of neurotrophic signaling pathways by polyphenols. Drug Des. Devel. Ther. 10, 23-42. doi:10.2147/DDDT.S96936

Nikov, G.N., Hopkins, N.E., Boue, S., Alworth, W.L., 2000. Interactions of dietary estrogens with human estrogen receptors and the effect on estrogen receptor-estrogen response element complex formation. Environ. Health Perspect. 108, 867-72. 
Pazaiti, A., Kontos, M., Fentiman, I.S., 2012. ZEN and the art of breast health maintenance. Int. J. Clin. Pract. 66, 28-36. doi:10.1111/j.1742-1241.2011.02805.x

Schoental, R., 1974. Role of Podophyllotoxin in the Bedding and Dietary Zearalenone on Incidence of Spontaneous Tumors in Laboratory Animals. Cancer Res. 34.

Seo, H.-S., Choi, H.-S., Kim, S.-R., Choi, Y.K., Woo, S.-M., Shin, I., Woo, J.-K., Park, S.-Y., Shin, Y.C., Ko, S.-G., Ko, S.-K., 2012. Apigenin induces apoptosis via extrinsic pathway, inducing p53 and inhibiting STAT3 and NFKB signaling in HER2-overexpressing breast cancer cells. Mol. Cell. Biochem. 366, 319-34. doi:10.1007/s11010-012-1310-2

Shier, W.T., Shier, A.C., Xie, W., Mirocha, C.J., 2001. Structure-activity relationships for human estrogenic activity in zearalenone mycotoxins. Toxicon 39, 1435-8.

Ström, A., Hartman, J., Foster, J.S., Kietz, S., Wimalasena, J., Gustafsson, J. -A., 2004. Estrogen receptor beta inhibits 17 beta-estradiol-stimulated proliferation of the breast cancer cell line T47D. Proc. Natl. Acad. Sci. U. S. A. 101, 1566-71. doi:10.1073/pnas.0308319100

Taupin, P., 2009. Apigenin and related compounds stimulate adult neurogenesis. Mars, Inc., the Salk Institute for Biological Studies: WO2008147483. Expert Opin. Ther. Pat. 19, 523-7. doi:10.1517/13543770902721279

Whitten, P.L., Patisaul, H.B., 2001. Cross-species and interassay comparisons of phytoestrogen action. Environ. Health Perspect. 5-20.

Zhao, J., Cheng, Y.-Y., Fan, W., Yang, C.-B., Ye, S.-F., Cui, W., Wei, W., Lao, L.-X., Cai, J., Han, Y.-F., Rong, J.-H., 2015. Botanical Drug Puerarin Coordinates with Nerve Growth Factor in the Regulation of Neuronal Survival and Neuritogenesis via Activating ERK1/2 and PI3K/Akt Signaling Pathways in the Neurite Extension Process. CNS Neurosci. Ther. 21, 61-70. doi:10.1111/cns.12334 


\section{Figure legends}

Table 1: Chemical class and structure of dietary estrogenic compounds used in this study.

Figure 1: Analysis of ER transactivation by the dietary compounds in breast cancer cell lines.

The expression of ER $\alpha$ and ER $\beta$ mRNA in MCF-7 and T47D cells was measured by real time PCR from total cDNA (A). Expression values were normalized to GAPDH mRNA levels and the re sults were expressed as relative expression of ER $\alpha$ found in each cell line from 8-10 independent experiments. MCF-7 cells (B), T47D cells (C) were transfected with an ERE-TK luciferase reporter plasmid and a CMV- $\beta$-galactosidase vector as a control for transfection efficiency. Then, cells were treated in serum-free medium with solvent (black) as the negative control and with various concentrations of E2 (blue), apigenin (Api) (green), liquiritigenin (Liq) (purple), daidzein (Dai) (grey), genistein (Gen) (brown), coumestrol (Cou) (pink), resveratrol (Res) (yellow) and zearalenone (Zea) (red). The results are expressed as percentage of luciferase activity obtained with E2 $10^{-9} \mathrm{M}$ treatment and are the mean of 3 independent experiments +/- SEM. * p-value $<0.05, * * p$-value $<0.01$ and $* *$ p-value $<$ 0.001 with one-way ANOVA and Bonferroni's post hoc test used to compare control cells with the other treatments.

Figure 2: Effect of the dietary compounds on the proliferation of breast cancer cell lines.

MCF-7 cells $(A, B, C)$ and T47D cells $(D, E, F)$ were grown for 6 days in serum-free medium in the presence of solvent as the negative control, with various concentrations of E2 (blue line) as the positive control, with flavones (A, D) (apigenin, green line and liquiritigenin, purple line), with isoflavones (B, E) (daidzein, gray line and genistein, brown line) or with other chemical classes (C, F) (coumestrol, pink line; resveratrol, yellow line and zearalenone, red line). Cell numbers were determined for each set of conditions. The results are expressed as percentage of the cell number obtained with E2 $10^{-9} \mathrm{M}$ and are the mean of 3 independent experiments $+/-$ SEM. * $p$-value $<0.05$ 
with one-way ANOVA and Bonferroni's post hoc test used to compare E2 treatment and the other treatments.

Figure 3: Effect of the dietary compounds on CXCL12 expression in MCF-7 and T47D cells.

MCF-7 cells (A) and T47D cells (B) were grown for $24 \mathrm{~h}$ in serum-free medium and then treated for 24 $\mathrm{h}$ with solvent as the negative control (black) and with various concentrations of E2 (blue), apigenin (green), liquiritigenin (purple), daidzein (grey), genistein (brown), coumestrol (pink), resveratrol (yellow) and zearalenone (red). The relative expression of CXCL12 was assessed by real time PCR and normalized using the expression of the housekeeping gene GAPDH. The results are expressed as percentage of CXCL12 relative expression in E2 $10^{-9} \mathrm{M}$ treated cells and are the mean +/- SEM of 3 to 4 independent experiments. ${ }^{*} p$-value $<0.05, * * p$-value $<0.01$ and $* * * p$-value $<0.001$ with oneway ANOVA and Bonferroni's post hoc test used to compare control cells with the other treatments.

Figure 4: Analysis of dietary compounds on ERa transactivation and differentiation of PC12-ER cells. Transcriptional activity of PC12-ER cells transfected with an ERE-TK luciferase reporter plasmid and a CMV- $\beta$-galactosidase vector as a control for transfection efficiency $(A)$. Then, cells were treated in serum-free medium and treated with solvent (black) as the control and E2 (blue) and with various concentrations of apigenin (green), liquiritigenin (purple), daidzein (grey), genistein (brown), coumestrol (pink), resveratrol (yellow) and zearalenone (red). The results are expressed as the percentage of luciferase activity obtained with E2 $10^{-9} \mathrm{M}$ treatment and are the mean of 3 independent experiments +/- SEM.

PC-12 ER (B) and PC12-Cont (B, cartridge) cells were treated for $72 \mathrm{~h}$ with NGF associated with solvent (control), E2 $10^{-9} \mathrm{M}$ or with NGF and various concentrations of apigenin (green), liquiritigenin (purple), daidzein (grey), genistein (brown), coumestrol (pink), resveratrol (yellow) and zearalenone (red). Then, cells were photographed and the percentage of cells bearing at least one ne urite was determined. The results are expressed as the percentage of NGF-treated cells bearing neurites and 
are the mean of at least 3 independent experiments +/- SEM. ${ }^{*} p$-value $<0.05,{ }^{* *} p$-value $<0.01$ and *** $p$-value $<0.001$, with one-way ANOVA and Bonferroni's post hoc test used to compare control cells with the other treatments. \#\#p-value $<0.01$ and \#\#\# p-value $<0.001$, with one-way ANOVA and Bonferroni's post hoc test used to compare E2-treated cells with the other treatments. 
Figure 1

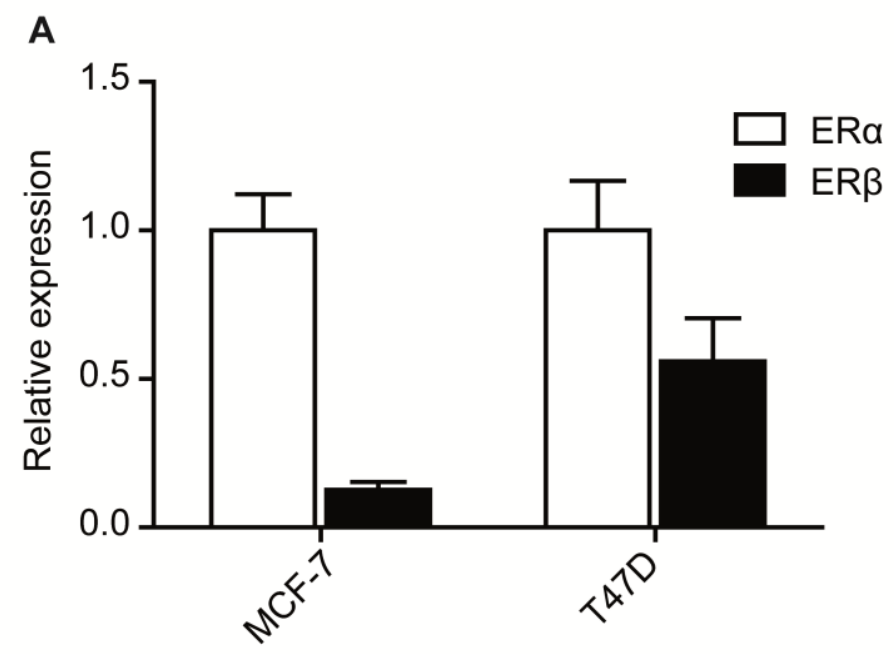

B
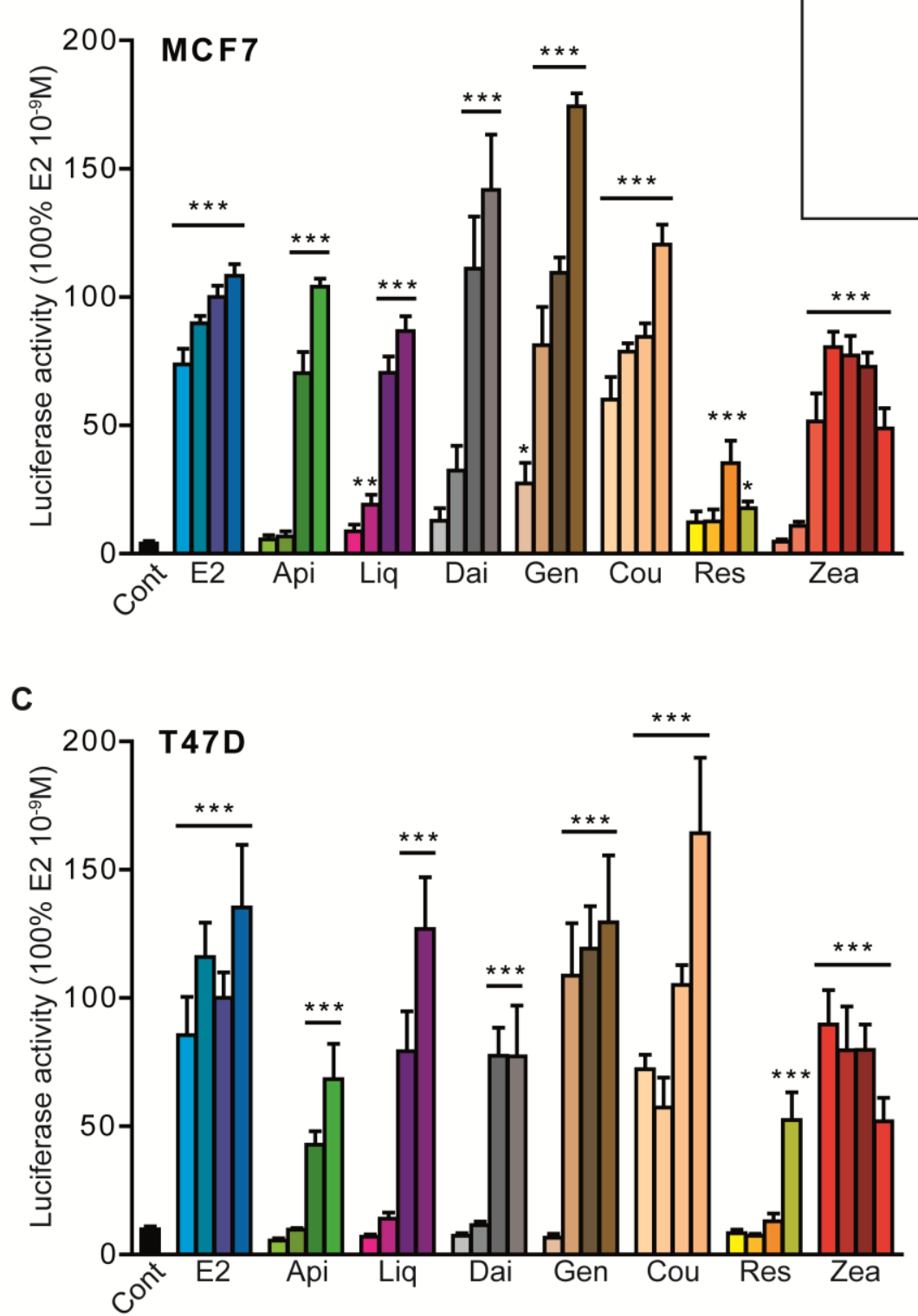
Figure 2
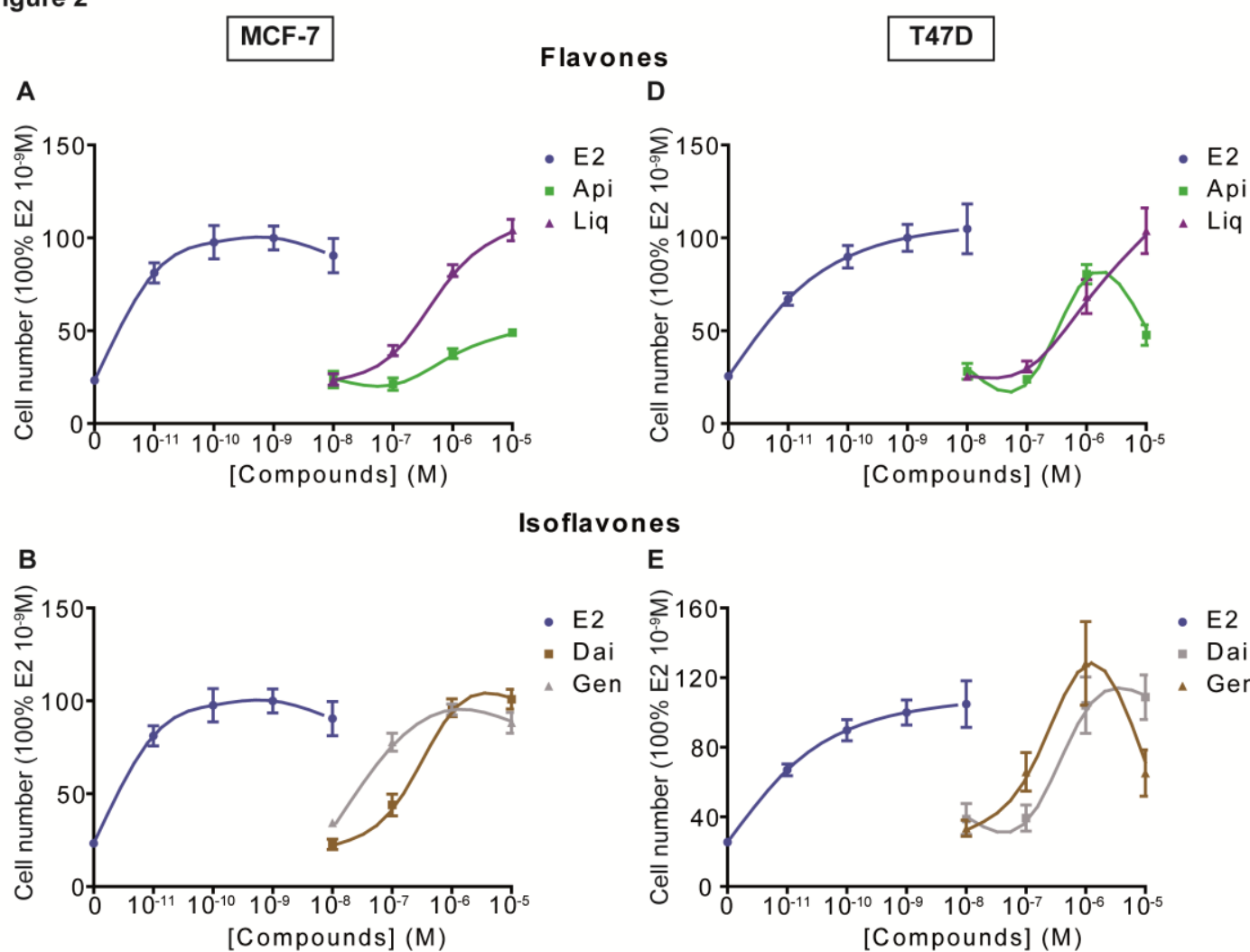

Isoflavones
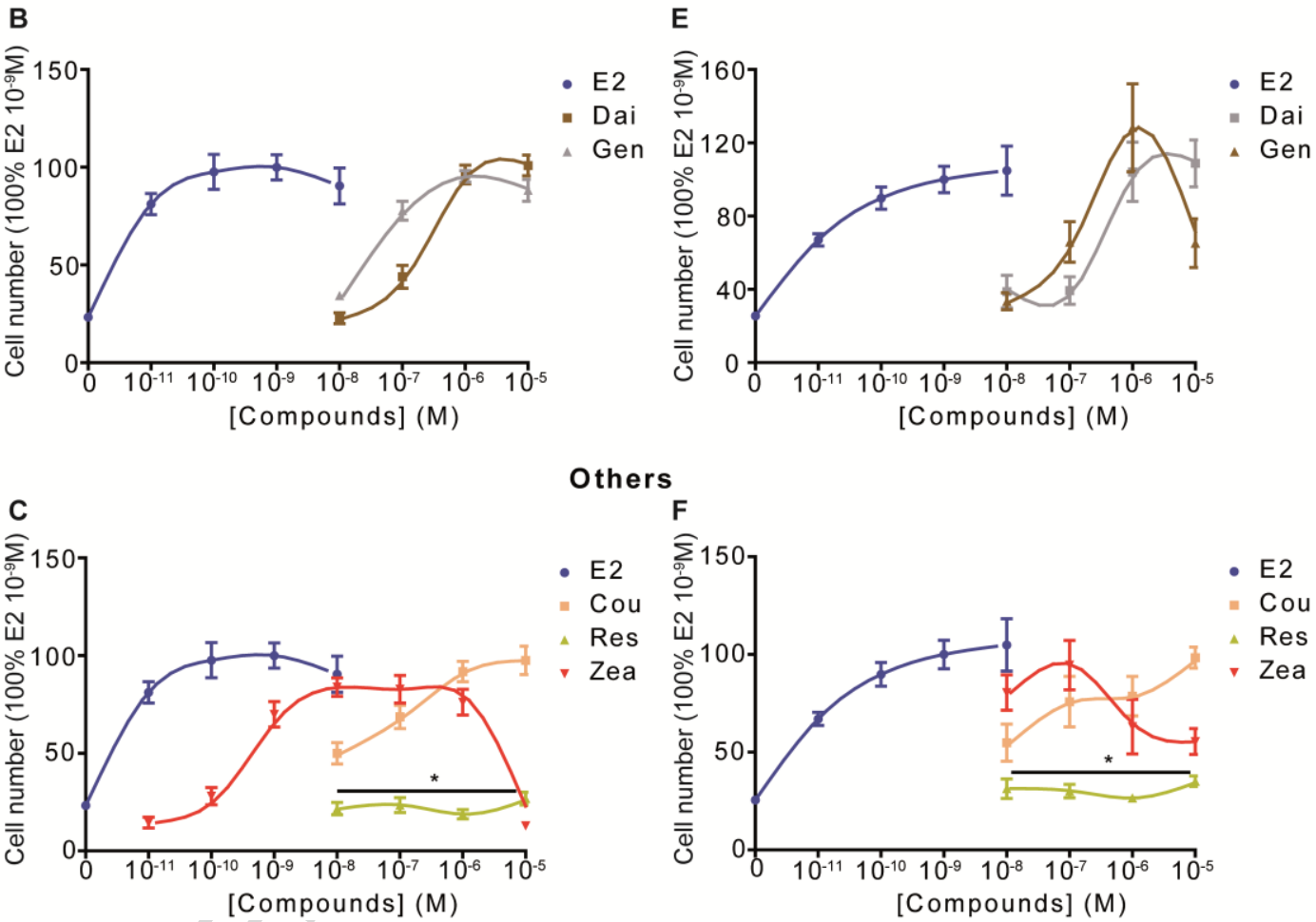

Others

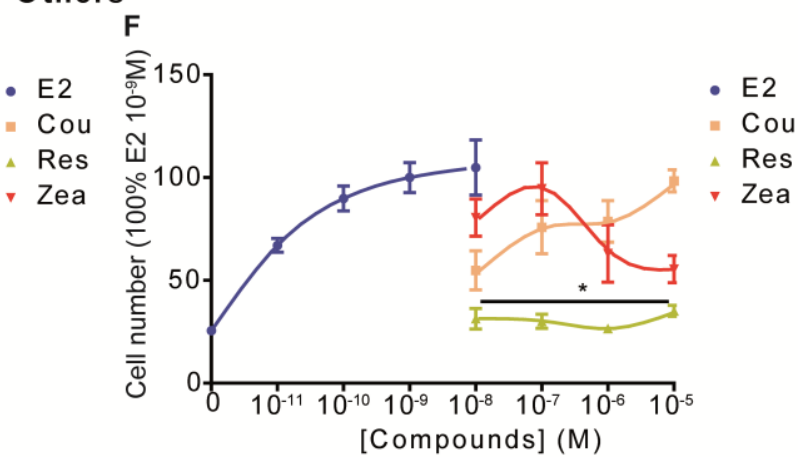


Figure 3

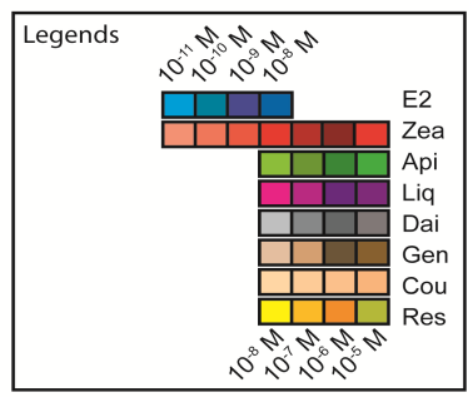

A

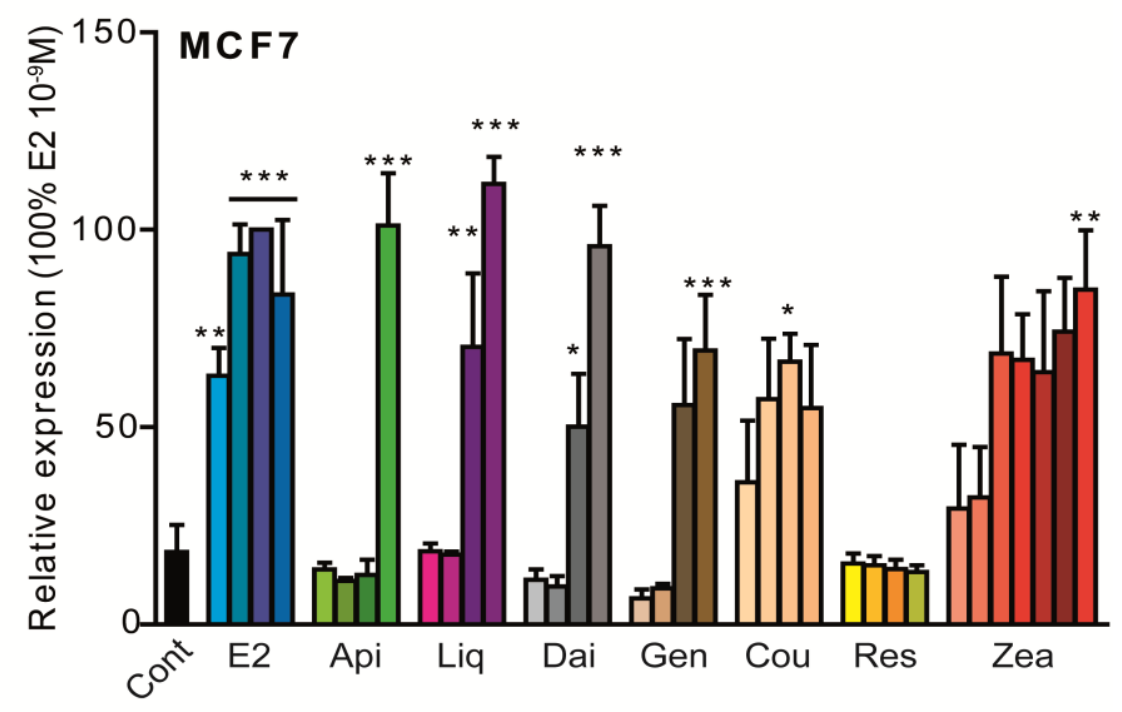

B

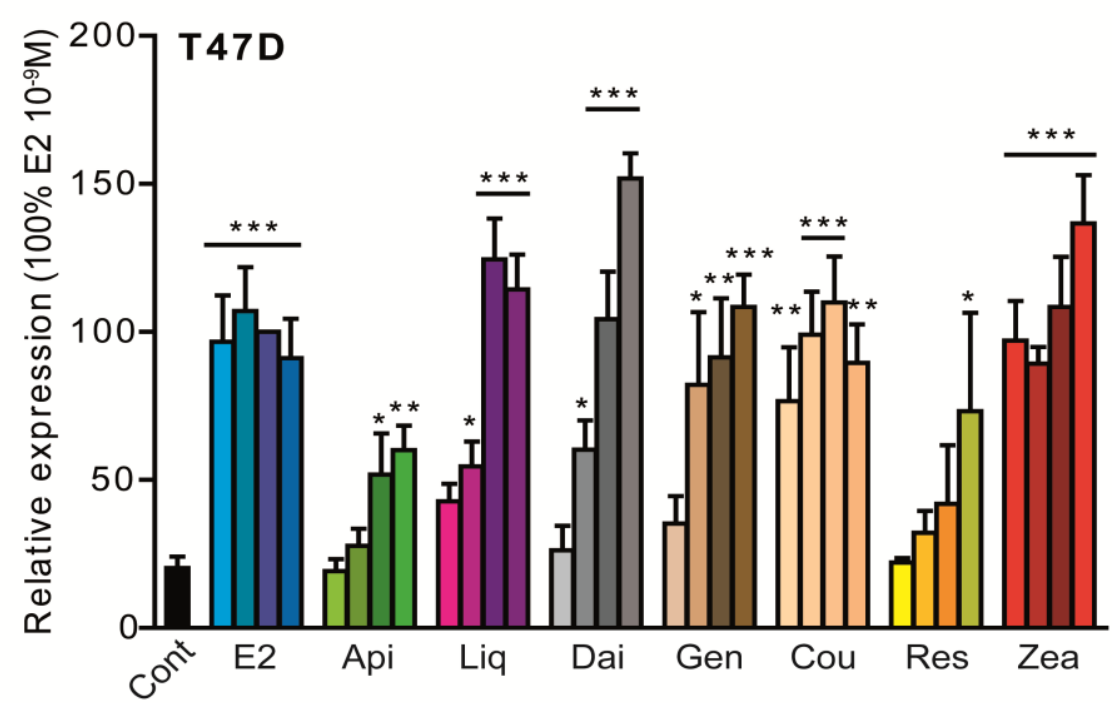


Figure 4

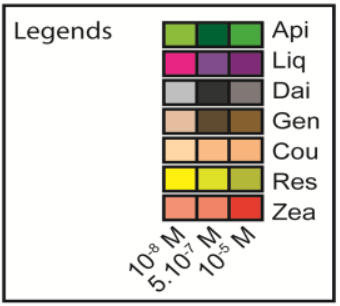

A
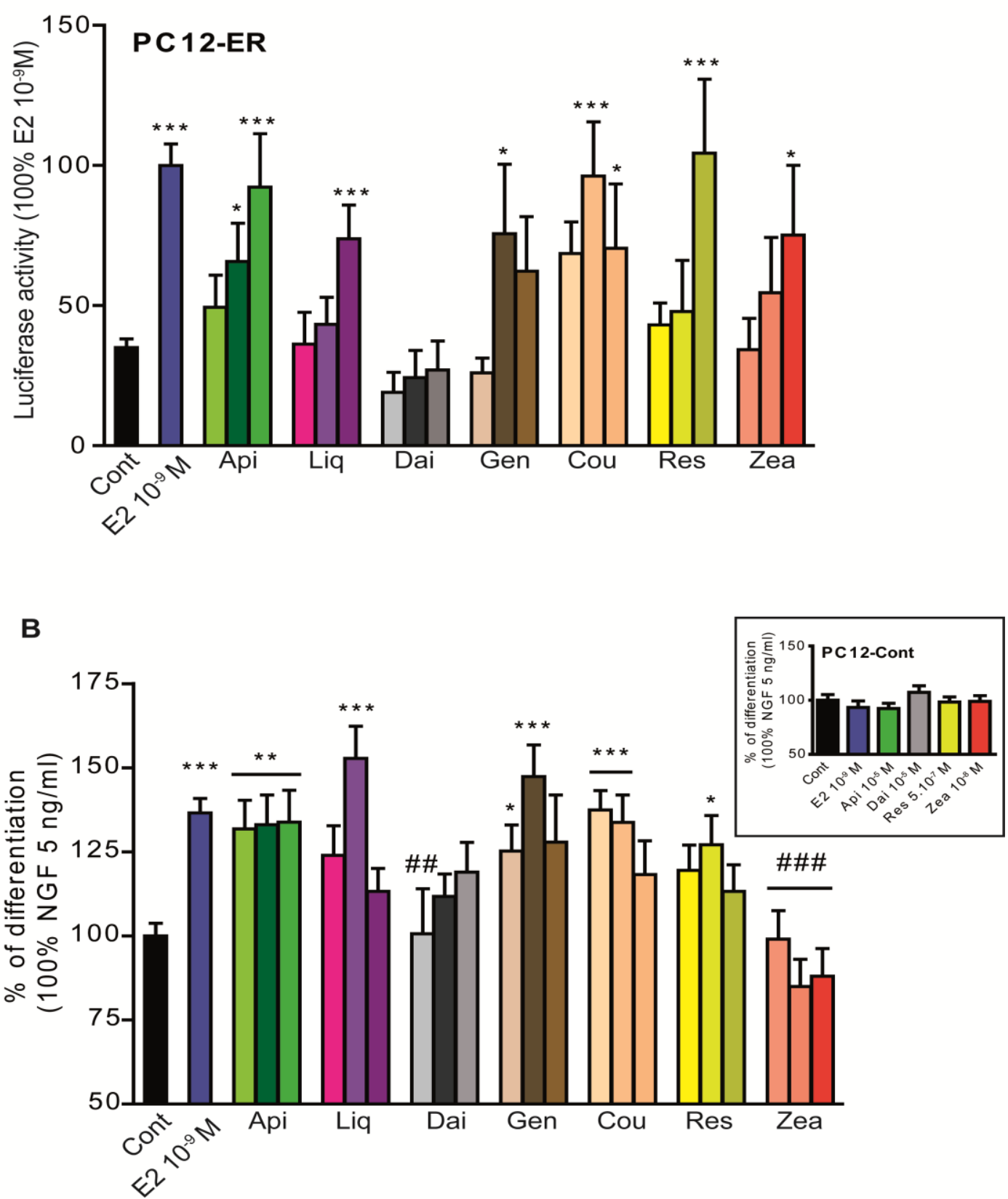
ACCEPTED MANUSCRIPT

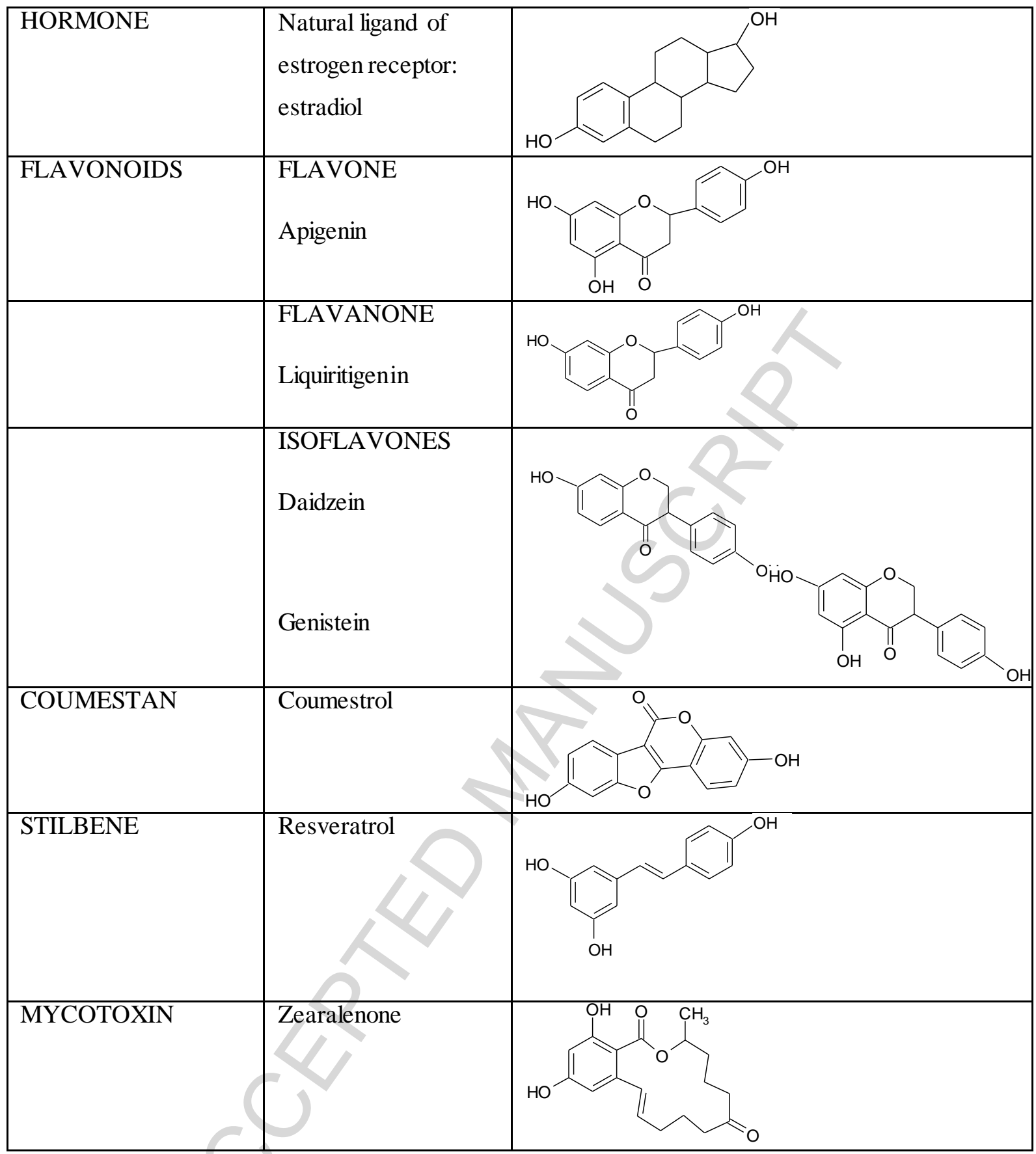

Table 1

26 


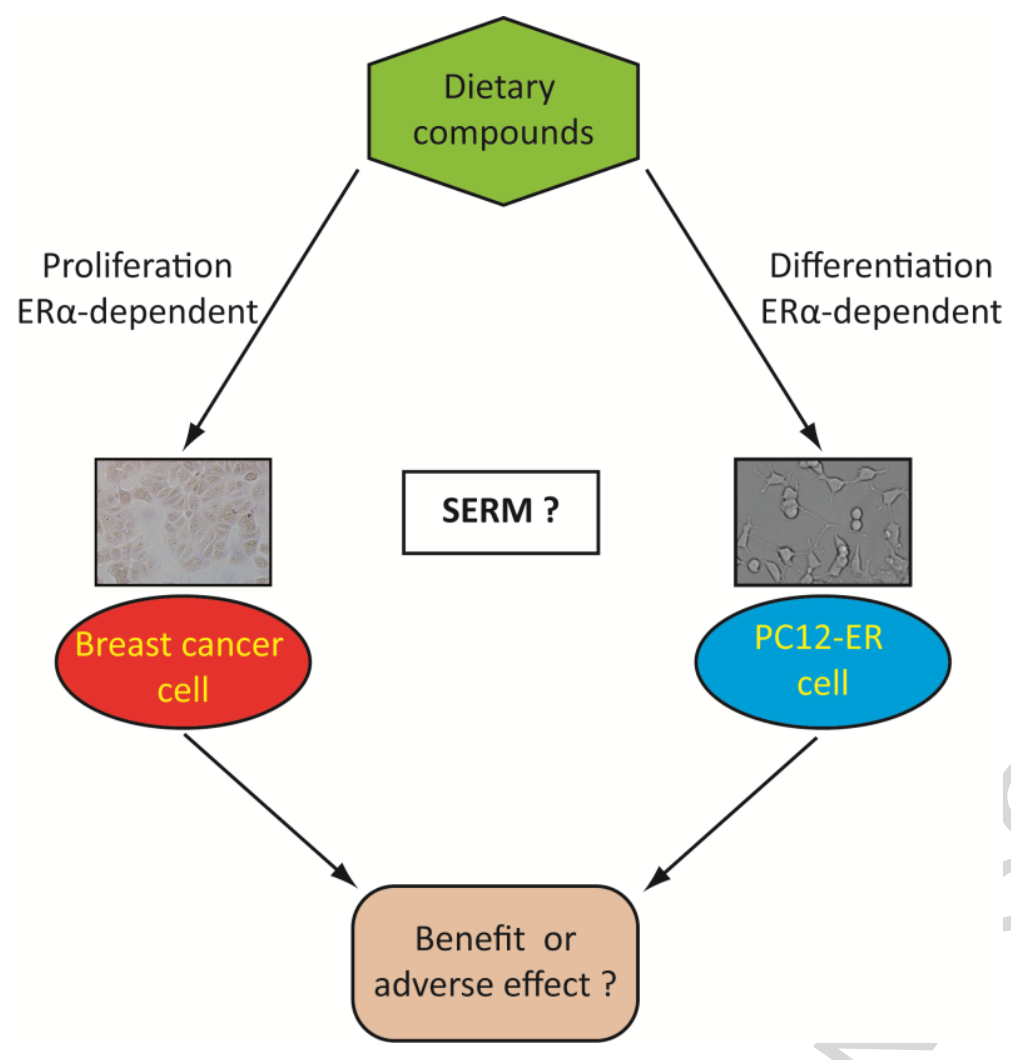

Graphical abstract 